\title{
Discovery of Novel Leishmanicidal Drugs with Potential L-type Calcium Channel Blockage, Designed by Similarity-Based Virtual Screening Approaches
}

\author{
Leonardo B. Federico ${ }^{1}$, Laura M. Alcântara ${ }^{3}$, Carolina B. Moraes ${ }^{2,3,4}$, \\ Lucio H. Freitas-Junior ${ }^{2,3,4}$, Joaquín M. C. Rosa ${ }^{5}$, Carlos H. T. P. Silva*1

\begin{abstract}
${ }^{1}$ School of Pharmaceutical Sciences of Ribeirão Preto, University of São Paulo, Ribeirão Preto, São Paulo, SP, Brazil ${ }^{2}$ Butantan Institute, São Paulo, SP, Brazil

${ }^{3}$ National Laboratory of Biosciences (LNBio) National Center for Research in Energy and Materials (CNPEM), Campinas, SP, Brazil

${ }^{4}$ Department of Microbiology, Institute of Biomedical Sciences, University of São Paulo, São Paulo, SP, Brazil

DDepartment of Pharmaceutical and Organic Chemistry, Faculty of Pharmacy, University of Granada,

University Campus of Cartuja CP 18071, Granada, Spain
\end{abstract}

Received Date: November 20, 2017 Accepted Date: December 22, 2017 Published Date: January 04, 2018

Citation: Federico LB, Alcântara LM, Moraes CB, Freitas LH, Rosa JMC, Carlos HTP Silva (2018). Discovery of Novel Leishmanicidal Drugs with Potential L-type Calcium Channel Blockage, Designed by Similarity-Based Virtual Screening Approaches. POJ Pharma Sci. 1(1): 1-6.

*Corresponding Author: Carlos H. T. P. Silva, PhD, Professor, School of Pharmaceutical Sciences of Ribeirão Preto, University of São Paulo, Ribeirão Preto, São Paulo, SP, Brazil, Tel: +55 163315 4717, Fax: +55 163602 4879;

Email: tomich@fcfrp.usp.br

\begin{abstract}
Calcium channel blockage as a therapeutic strategy for treatment of leishmaniasis has demonstrated a promising pathway of treatment for this pathology. In this work, virtual screening approaches have been performed in several databases of commercially available compounds in order to design novel leishmanicidal compounds with potential L-type calcium channel blockage. Several compounds were tested against Leishmania intracellular parasites, using High-Content Analyses Assays. Results have indicated two compounds with new chemotypes, which were able to kill the parasite, with moderate activity and high efficacy, without toxicity for the host. Compounds here discovered represent a very useful molecular simplification compared to the reference or control compound, amphotericin B, with suitable ADME/Tox properties and synthetic accessibility.
\end{abstract}

Keywords: Leishmaniasis, L-type Calcium Channel Blockers, High-Content Analysis, Similarity-Based Virtual Screening, Structure-Based Virtual Screening

Leishmaniasis is a parasitic and lethal disease caused by hemoflagellate parasites of the genus Leishmania spp. This disease affects mainly low-income and developing countries and stands out because it is endemic in 98 countries, 3 territories and 5 continents, for reaching about 2 million annual cases and leaving more than 350 million people at risk [1]. The life cycle of this parasite is heterotoxene, which has two types of hosts - a vertebrate and another invertebrate, and its vector is the female of the mosquito Lutzomyia longipalpis. Leishmaniasis is transmitted through the bite of the female sand-fly contaminated with the promastigotes forms of the disease, which rapidly transform into amastigote forms after invading the reticuloendothelial cells of the host. In macrophages, the amastigote forms actively reproduce until they cause tissue rupture, a hallmark of the disease. The amastigote form serves as an infecting stage for the female sand-fly that turns into promastigote in its midgut and migrates into the salivary gland, ready to infect a new host by closing the life cycle of the parasite [2-5].

Copyright: (C2018 Carlos HTP Silva, et al. This is an open-access article distributed under the terms of the Creative Commons Attribution License, which permits unrestricted use, distribution, and reproduction in any medium, provided the original author and source are credited. 
The current treatment for leishmaniasis is the same one used since the 1940s. The drugs of first choice are antimoniate$\mathrm{N}$-methylglucine (Glucantime ${ }^{\circledR}$ ), sodium stibogluconate (Pentostam ${ }^{\circledR}$ ) and amphotericin B. It is noteworthy that such drugs have potential cardiac, hepatic, pancreatic and renal toxicity [6]. The ability of the parasite to acquire resistance to these drugs is another aggravating factor to the traditional treatment, which prevents the action of these drugs $[3,7]$. Therefore, thus, research for new drugs or strategies capable of preventing the progression of this disease becomes increasingly relevant.

Drugs that act via calcium channel blockage point out as a viable alternative for combating leishmaniasis, since they prevent the adhesion of Leishmania species to the macrophage, in the early stages of the disease, thus allowing the progression of the disease.[8] Reported calcium channel blockers include amlodipine, nimodipine, lacidipine, 1,4-dihydropyridine (verapamil and derivatives), which can be used as prototypes for investigation of new chemical entities capable of conferring leishmanicidal action [9-11].

In vitro studies reported for nimodipine indicate a $50 \%$ inhibitory concentration values of 81.2 and $21.5 \mu \mathrm{M}$ against respectively promastigotes and intracellular amastigotes of Leishmania (L.) chagasi, and IC50 concentration range between 31 and $128 \mu \mathrm{M}$ from Leishmania (L.) amazonensis and Leishmania (L.) major [11]. Actually, these reported concentrations could be out of the range found to have significant cardiovascular pharmacology, which implies in dissociation of these two distinct pharmacological actions.

Computational chemistry approaches were here used to identify new potential calcium channel inhibitors with leishmanicidal action. Initially were designed and selected nine hits with potential leishmanicidal activity, and four of them have shown activity identified after assays with cell cultures. Two compounds were detected with promising leishmanicidal activity and efficacy as well.

Design of such compounds was based on virtual screening approaches (Figure 1) [12]. Virtual screening was initially performing in six commercial compounds databases based on similarity (shape and electrostatics) of reported active ones. For this step, a dihydropyridine was used as template and the ROCS and EON softwares were used to access shape and electrostatic similarities, respectively [13-15].

Figure 1. Flowchart summarizing the structure- and similarity-based virtual screening approaches used.

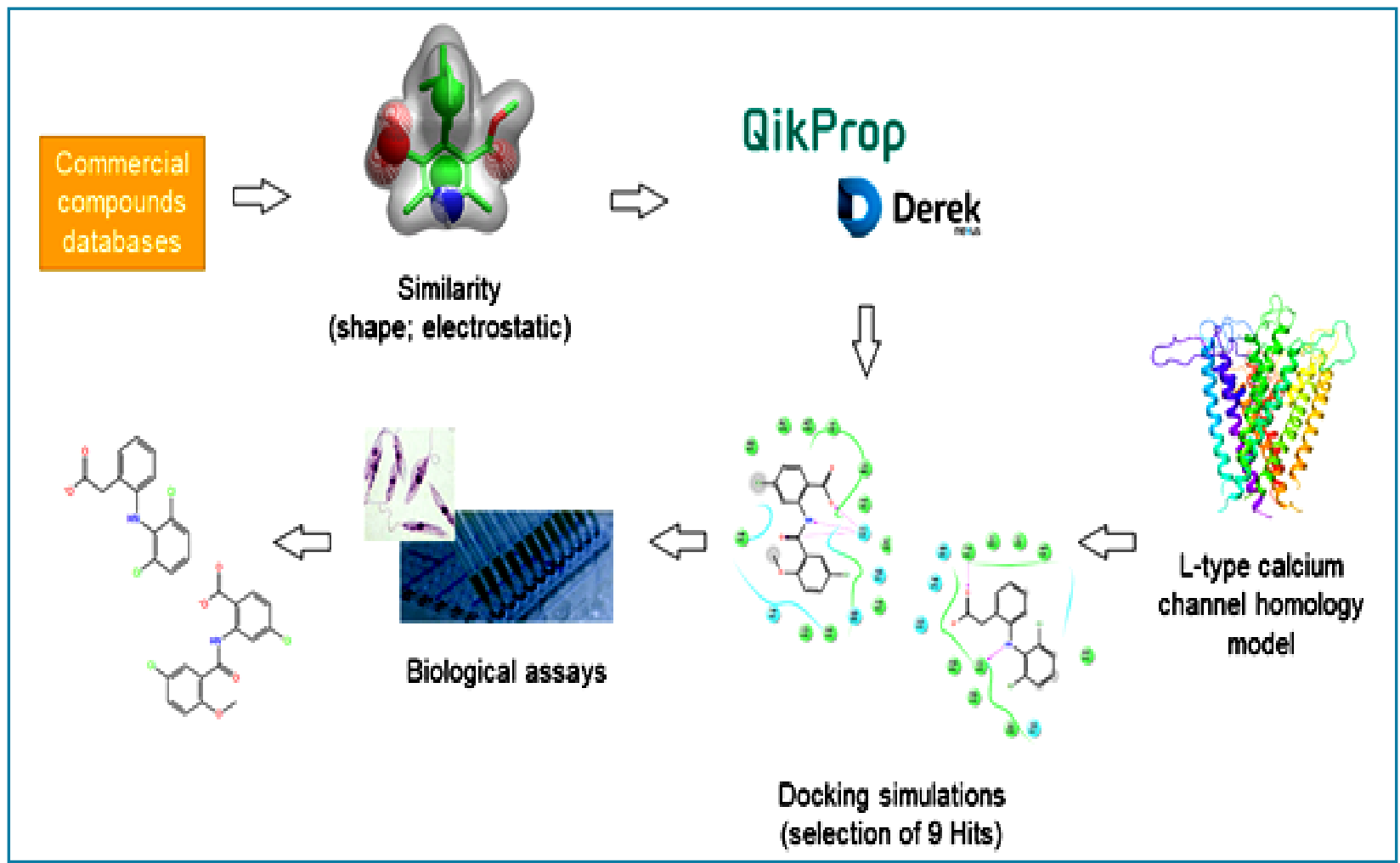

The ROCS and EON softwares from the OpenEye Company were used in the LBDD studies. ROCS sorts compounds by comparing the conformation and volume of a template compound (reference) with the conformation and volume of compounds from the databases, whereas EON uses electrostatic similarity to screen compounds previously selected with ROCS.
Compounds thus selected had their pharmacokinetics and toxicological properties in silico investigated, using the QikProp and DEREK softwares, respectively $[16,17]$. The QikProp software, from Schrodinger company makes the prediction of selected ADME properties, based on a 95\% range of known drugs. DEREK is a knowledge-based molecular toxicity prediction

Citation: Federico LB, Alcântara LM, Moraes CB, Freitas LH, Rosa JMC, Carlos HTP Silva (2018). Discovery of Novel Leishmanicidal Drugs with Potential L-type Calcium Channel Blockage, Designed by Similarity-Based Virtual Screening Approaches.

POJ Pharma Sci. 1(1): 1-6. 
software. Using DEREK, the medicinal chemist can analyze the structure of a query molecule, looking for possible toxicophoric groups and its toxicological risks, using rules of bases.

The "survivor" compounds with suitable ADME/Tox properties were then analyzed by visual inspection, using the Maestro software, about the binding modes inside a calcium channel structure, thus proposed by docking approaches [18]. Since there are no reported leishmanicidal drugs so far with potential L-type calcium channel blockage and designed by similaritybased virtual screening approaches, since no calcium channel structures are deposited in the Protein Data Base (PDB), it was necessary to build an L-type calcium channel structure using homology modelling. The comparative or homology modelling approach was used, and the model thus obtained and posteriorly used in the virtual screening experiments was previously evaluated and validated regarding stereochemistry, atomic contacts and chemical environment as well.

Docking simulations were carried out to propose reliable binding modes for all the compounds into two main regions of the calcium channel: the central pore and the allosteric site. In order to obtain a more reliable result, we used two different and well evaluated flexible docking approaches (using the Glide and GOLD softwares) and the results here obtained were compared by visual inspection of the respective docking poses $[19,20]$.

Visual inspection of the binding poses proposed by docking for the selected compounds inside the two regions of the calcium channel was based on the potential interactions that such compounds could stablish with conserved amino acid residues, reported in literature as the binding residues of the calcium channel. Finally, nine hits were thus selected: compounds 1 (ZINC7563), 2 (ChemBridge7991096), 3 (ZINC03876174), 4 (BindingDB28699), 5 (MolPort 000-900-772), 6 (ZINC 1281), 7 (ZINC125031), 8 (MolPort 001-579-125) and 9 (MolPort 002305-206), with promising calcium channel blocking ability, where two of them showed interesting leishmanicidal activity and efficacy as well (Figure 2).

Figure 2. 2D representation of the nine compounds here investigated and selected with potential calcium channel blocking activity, using similarity-based virtual screening approaches. The last four compounds (6 to 9) were tested against Leishmania intracellular parasites, using High-Content Analysis Assays.

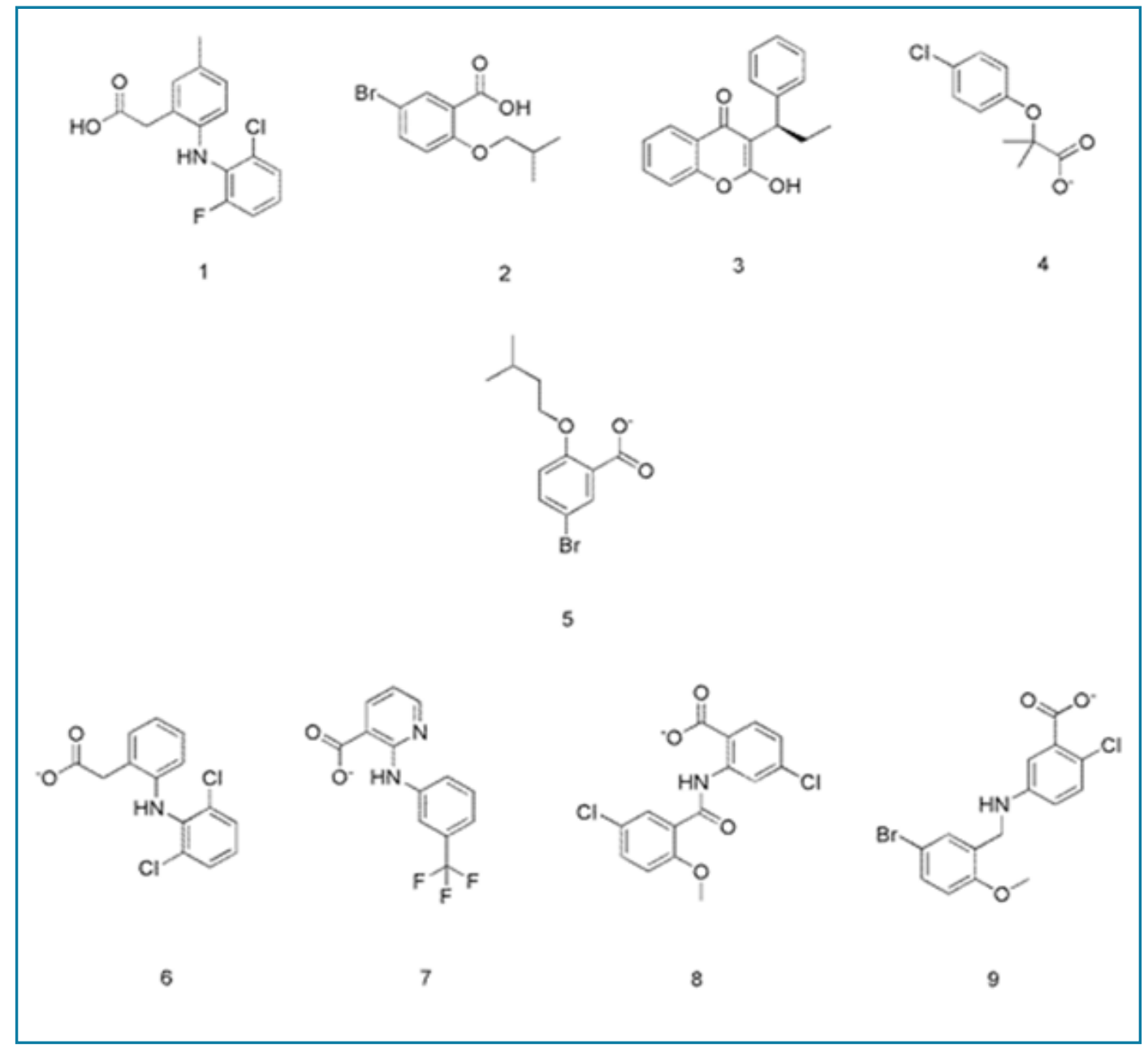

Citation: Federico LB, Alcântara LM, Moraes CB, Freitas LH, Rosa JMC, Carlos HTP Silva (2018). Discovery of Novel Leishmanicidal Drugs with Potential L-type Calcium Channel Blockage, Designed by Similarity-Based Virtual Screening Approaches. POJ Pharma Sci. 1(1): 1-6. 
A high content screening assay was performed for assessing compounds' activity against L. infantum intracellular amastigotes. The use of the high content screening approach for image processing and data quantification provides a multiparametric assessment of biological model, which is more unbiased, sensitive and reliable, if compared to classical manual counting and fluorescence-based methodologies. THP-1 was plated onto 384-well plates in RMPI complete media containing PMA, and incubated for $48 \mathrm{~h}$ for complete differentiation into macrophage-like cells. Then, L. infantum promastigotes (enriched for metacyclic forms) were added (50 parasites/ cell). After $24 \mathrm{~h}$ of infection, negative controls (0.5\% DMSO), positive controls (10 $\mu \mathrm{M}$ amphotericin B) and compounds in a twofold dilution series (from 100 to $0.1 \mu \mathrm{M}$ ) were added into the plate. After $48 \mathrm{~h}$ of drug exposure, plates were fixed with
4\% PFA, stained with $5 \mu \mathrm{M}$ Draq 5 and processed for image analysis. The multiparametric high content analysis yielded the quantification of host cells number, infection ratio and number of amastigotes per infected cells. Activity values were processed with the Graphpad Prism software, for generation of sigmoidal dose-response (variable slope) nonlinear curve fitting and determination of EC50 (half-maximum effective concentration, i.e., the concentration of compound that causes a $50 \%$ reduction of infection in comparison to non-treated infected controls) and CC50 (50\% reduction in cell number, when compared to nontreated infected controls) values by interpolation. The selectivity index (SI) was calculated by dividing CC50/EC50. When CC50 could not be determined, the SI was estimated as being greater than the highest compound concentration tested divided by the EC50. Data are presented on Table 1.

Table 1. Biological activity of selected compounds against Leishmania infantum intracellular amastigotes

\begin{tabular}{|c|c|c|c|c|}
\hline Compound & $\mathrm{EC}_{50}(\mu \mathrm{M})$ & $\mathrm{CC}_{50}(\mu \mathrm{M})$ & S.I (H) & Max. act. (\%) \\
\hline Amphotericin B & $1.5 \pm 0.1$ & $22.2 \pm 2.3$ & 15.2 & $99.0 \pm 0.1$ \\
\hline 7 & nd & nd & - & $48.4 \pm 9.6$ \\
\hline & $51.9 \pm 7.4$ & nd & $>1.9$ & $95.5 \pm 5.0$ \\
\hline 6 & & & & \\
\hline & $43.0 \pm 2.9$ & nd & $>2.3$ & $90.6 \pm 7.3$ \\
\hline & nd & nd & - & $22.3 \pm 5.8$ \\
\hline 9 & & & & \\
\hline
\end{tabular}

EC50 (concentration of compound that causes $50 \%$ reduction in infection rates) and CC50 (concentration of compound that causes $50 \%$ reduction in host cell number) values are expressed as averages \pm standard deviations; nd, not determined; Max. Act.: maximum activity observed in the dose response- curve. S.I. selectivity index.

Citation: Federico LB, Alcântara LM, Moraes CB, Freitas LH, Rosa JMC, Carlos HTP Silva (2018). Discovery of Novel Leishmanicidal Drugs with Potential L-type Calcium Channel Blockage, Designed by Similarity-Based Virtual Screening Approaches. 
Among the tested molecules, compounds 6 and 8 presented high efficacy, leading to an infection inhibition of 95.5 and $90.6 \%$, respectively. Furthermore, these molecules had relatively moderate potency against intracellular amastigotes (EC50 = 51.9 and $43.0 \mu \mathrm{M}$, respectively), compared to the reference compound amphotericin B $(E C 50=1.5 \mu \mathrm{M})$. Additionally, no toxicity was observed against the host cell THP-1.

Although these compounds do not show EC50 values comparable to that of the reference compound, the results obtained for at least two compounds are interesting from a structural point of view. Such compounds show an interesting molecular simplification when compared to amphotericin B, with suitable synthetic accessibility and a large potential of derivatization, besides potential proper ADME/Tox properties. In fact, no toxicity was observed in vitro for such compounds against the host cell THP-1. Finally, we are now proceeding with lead optimization of the compounds in order to improve both pharmacodynamic and pharmacokinetic properties, in the search for calcium channel blockers as future leishmanicidal drugs. Also, an additional positive control using calcium channel blocker, particularly containing a dihydropyridine scaffold, will be used in our further experiments.

\section{Acknowledgements}

The authors acknowledge CNPq (Conselho Nacional de Desenvolvimento Científico e Tecnológico), in Brazil, for financial support.

\section{Conflict of Interest}

The authors declare no conflicts of interest with respect to the content, authorship, and/or publication of this article.

\section{References}

1. World Health Organization. Control of the leishmaniases. World Health Organ Tech Rep Ser. 2010;949:1-186.

2. Zeibig E. Clinical Parasitology: A Practical Approach. 2nd ed. Brazil: Elsevier Health Sciences; 2014.

3. Mahajan RC, Mohan K. Epidemiology of visceral leishmaniasis and its control. In: Ozcel MA, Alkan MZ, editors. Parasitology for the 21st Century. Oxon, UK: Cab International; 1996. p. 41-9.

4. David CV, Craft N. Cutaneous and mucocutaneous leishmaniasis. Dermatol Ther. 2009;22(6):491-502.

5. Vermelho AB, Supuran CT, Cardoso V, et al. Leishmaniasis : Possible New Strategies for Treatment. In: David M. Claborn, editor. Leishmaniasis - Trends in Epidemiology, Diagnosis and Treatment. Rijeka, Croatia: InTech; 2014. p. 352-376.

6. Ministry of Health. Secretariat of Health Surveillance. Department of Epidemiological Surveillance. Atlas of American
Integumentary Leishmaniasis. Clinical and Differential Diagnoses. 1st ed. Brasília - DF: Editora MS; 2006. p. 136.

7. Croft SL, Sundar S, Fairlamb AH. Drug Resistance in Leishmaniasis. Clin Microbiol Rev. 2006;19(1):111-126.

8. Misra S, Naskar K, Sarkar D, Ghosh DK. Role of Ca2+ ion on Leishmania-macrophage attachment. Mol Cell Biochem. 1991;102(1):13-18.

9. Palit P, Ali N. Oral therapy with amlodipine and lacidipine, 1,4-dihydropyridine derivatives showing activity against experimental visceral leishmaniasis. Antimicrob Agents Chemother. 2008;52(1):374-377.

10. Reimão JQ, Mesquita JT, Ferreira DD, Tempone AG. Investigation of Calcium Channel Blockers as Antiprotozoal Agents and Their Interference in the Metabolism of Leishmania (L.) infantum. Evid Based Complement Alternat Med. 2016;2016:1-9.

11. Tempone AG, Taniwaki NN, Reimão JQ. Antileishmanial activity and ultrastructural alterations of Leishmania (L.) chagasi treated with the calcium channel blocker nimodipine. Parasitol Res. 2009;105(2):499-505.

12. Federico LB, Santos CBR, Lobato CC, Gomes JS, Rosa JMC, Silva CHTP. Ligand- and Structure-Based Drug Design of Novel Calcium Channel Blockers. J Comput Theor Nanosci. 2017;14(7):3489-3502.

13. ROCS version 3.2.2.2. OpenEye Scientific Software. Santa Fe, NM, USA; 2017. Available from: http://www.eyesopen.com

14. Tresadern G, Bemporad D, Howe T. A comparison of ligand based virtual screening methods and application to corticotropin releasing factor 1 receptor. J Mol Graph Model. 2009;27(8):860-870.

15. EON version 2.2.0.5. OpenEye Scientific Software. Santa Fe, NM, USA; 2013. Available from: http://www.eyesopen.com

16. Small-Molecule Drug Discovery Suite 2015-2: QikProp, Schrödinger, LLC, New York, NY, 2015.

17. Marchant CA. Prediction of rodent carcinogenicity using the DEREK system for 30 chemicals currently being tested by the National Toxicology Program. Environ Health Perspect. 1996;104(suppl 5):1065-1073.

18. Schrödinger Release 2015-2: Maestro, Schrödinger, LLC, New York, NY, 2015.

19. Chen Z, Li H, Zhang QJ, et al. Pharmacophore-based virtual screening versus docking-based virtual screening: a benchmark comparison against eight targets. Acta Pharmacol

Citation: Federico LB, Alcântara LM, Moraes CB, Freitas LH, Rosa JMC, Carlos HTP Silva (2018). Discovery of Novel Leishmanicidal Drugs with Potential L-type Calcium Channel Blockage, Designed by Similarity-Based Virtual Screening Approaches.

POJ Pharma Sci. 1(1): 1-6. 
Sin. 2009;30(12):1694-1708.

20. Cole JC, Nissink JWM, Taylor R. Protein-Ligand Docking and Virtual Screening with GOLD. In: Shoichet B, Alvarez J, editors. Virtual Screening in Drug Discovery. Boca Raton, Florida, USA: Taylor \& Francis CRC Press; 2005. p. 379-415. Drugs with Potential L-type Calcium Channel Blockage, Designed by Similarity-Based Virtual Screening Approaches. POJ Pharma Sci. 1(1): 1-6. 